\title{
Resonance production from jet fragmentation
}

\author{
Christina Markert ${ }^{\mathrm{a}}$ \\ for the STAR Collaboration
}

University of Texas, Austin, TX 78712, USA

Received: 7 October 2008 / Revised: 28 January 2009 / Published online: 3 March 2009

(C) Springer-Verlag / Società Italiana di Fisica 2009

\begin{abstract}
Short lived resonances are sensitive to the medium properties in heavy-ion collisions. Heavy hadrons have larger probability to be produced within the quark gluon plasma phase due to their short formation times. Therefore heavy mass resonances are more likely to be affected by the medium, and the identification of early produced resonances from jet fragmentation might be a viable option to study chirality. The high momentum resonances on the away-side of a triggered di-jet are likely to be the most modified by the partonic or early hadronic medium. We will discuss first results of triggered hadron-resonance correlations in $\mathrm{Cu}+\mathrm{Cu}$ heavy ion collisions.
\end{abstract}

\section{Introduction}

Chiral symmetry restoration is one of the fundamental features of QCD at high temperatures and densities of nuclear matter which should occur around the phase transition $(0.8-$ $1.2 T_{\mathrm{c}}$ ) according to lattice QCD calculations [1]. The signature of chiral symmetry restoration could be mass shifts and/or broadenings of the hadronic resonance states inside the medium created in a heavy ion collision. Resonances from jet fragmentation can be produced early, depending on their hadronic formation times and might be medium modified through interactions with either the late partonic $\left(1.2 T_{\mathrm{c}}\right)$ or early hadronic $\left(0.8 T_{\mathrm{c}}\right)$ medium. To measure this effect, resonances also need to decay inside the medium. The formation time of hadrons from jet fragmentation depends on their mass and momentum. I will discuss the relevant momentum range for different resonances. Furthermore one needs to select the resonances on the away-side of a surface biased (triggered) jet in order to maximize their in-medium interactions. This will be discussed in Sect. 3 .

a e-mail: cmarkert@physics.utexas.edu

\section{Formation time}

The formation time of hadrons and resonances from jet fragmentation can be calculated in a quantum mechanical approach $[2,3]$. An alternative approach, based on string fragmentation [4], arrives at a similar conclusion for heavy mass hadrons, light quark objects. A heavier hadron mass leads to a shorter formation time while a higher momentum causes a longer formation time. In the theoretical calculations [3] the probability of high momentum heavy hadron (or resonance) formation in the partonic medium is finite. Therefore we expect the resonance to be medium modified, after its formation, through parton-resonance interactions in the so called mixed degree of freedom phase of partons and hadrons. The proper momentum range needs to be defined by a lower and an upper limit to ensure the formation and decay of the resonance inside the chiral medium. If the width of the resonances is broadened in the medium their lifetimes is shortened which will increase the probability of the resonances decaying inside the medium. This would also result in a broader signal width in the invariant mass spectrum which decreases the statistical significance of the signal. Figure 2.1 shows the linear dependence of the average formation time on the transverse momentum of the hadron (or resonance) integrated over the fragmentation distribution of all produced jets at RHIC. The yellow shaded area is the lifetime of the QGP calculated by assuming a longitudinal Bjorken expansion with $T_{\mathrm{c}} \approx 180 \mathrm{MeV}$ for the critical temperature. The estimated lifetime at RHIC is $\tau_{\mathrm{QGP}}=6.2 \mathrm{fm} / \mathrm{c}$ and at the LHC is $\tau_{\mathrm{QGP}}=14 \mathrm{fm} / c$ based on an longitudinal Bjorken expansion [3]. The RHIC QGP lifetime is in agreement with the partonic lifetime, derived from the resonance suppression in the hadronic medium [5-9] and the pion HBT source lifetime measurement $(\Delta \tau=5-12 \mathrm{fm} / \mathrm{c})$ [10]. Figure 2.2 shows the corresponding linear dependence of the mean formation time on the transverse momentum of the hadron (or resonance) for the LHC. 


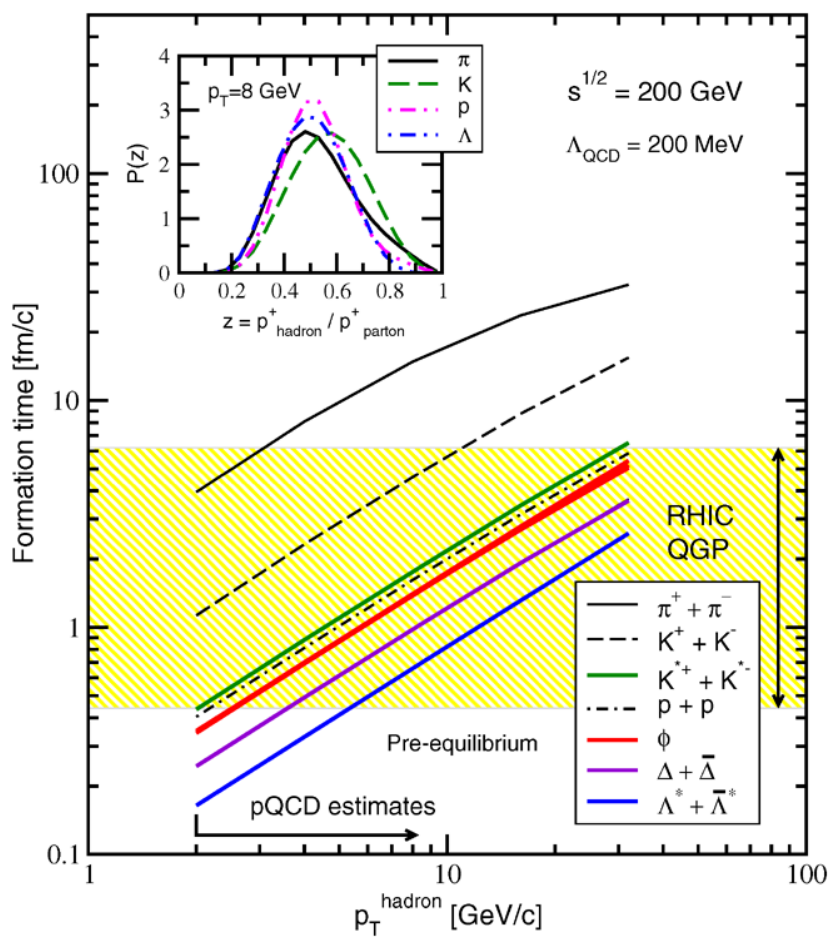

Fig. 2.1 Transverse momentum dependence of leading hadron formation times at RHIC energies, $\sqrt{s}=200 \mathrm{GeV}$. Results for both 'stable' particles and resonances are presented. The shaded area represents the estimated partonic lifetime at RHIC. Insert shows the meson and baryon distributions $P(z)$ at a fixed hadron momentum $p_{T}=8 \mathrm{GeV} / c$. The shaded area represents the estimated partonic lifetime at LHC using a Bjorken expansion [3]

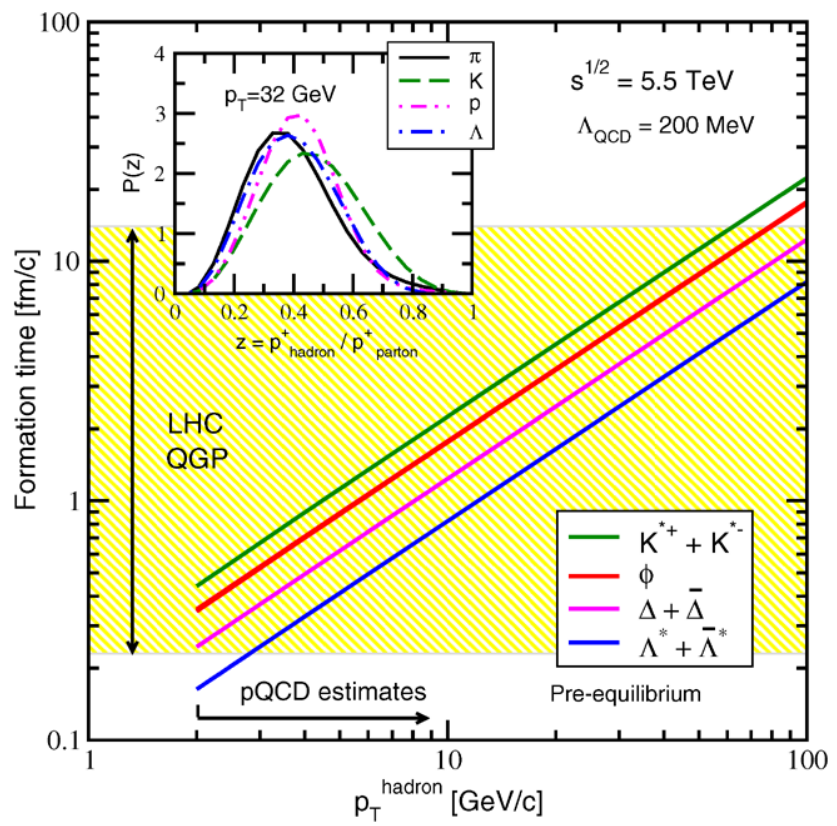

Fig. 2.2 Transverse momentum dependence of heavy resonance formation times at LHC energies, $\sqrt{s}=5.5 \mathrm{TeV}$. Insert shows the meson and baryon distributions $P(z)$ at a fixed hadron momentum $p_{T}=32 \mathrm{GeV} / c$. The shaded area represents the estimated partonic lifetime at LHC using a Bjorken expansion [3]

\section{Resonances in jets}

In order to extract the chirally restored resonances from the partonic and early hadronic medium, a correlation analysis using a jet or hadron triggered high momentum resonance is suggested. This also ensures that the resonance signal is largely unaffected by the late hadronic medium where regeneration of resonances will dilute it. UrQMD calculations suggest that the regeneration of resonances is predominant in the low momentum region $\left(p_{\mathrm{T}}<2 \mathrm{GeV} / c\right)$ [11], the study of chiral symmetry restoration is only suitable in the momentum region of $p_{\mathrm{T}}>2 \mathrm{GeV} / c$. High momentum resonances are less affected by the re-scattering and regeneration in the hadronic phase because the system moves with a smaller collective velocity than the probe, and thus the high momentum decay particles can escape. Resonances from jets where the hard scattering occurs near the surface are in particular unaffected by the medium. Thus the hadronic resonances from the same side jet in a triggered di-jet event will serve as the de facto in-vacuum reference for the medium modification measurement. The away-side distribution of jet resonances is selected to study chirality. Resonances in this distribution are likely to experience a longer path length in the medium. However their momenta should be large enough to be unaffected by re-scattering and regeneration in the subsequently produced hadronic medium. This idea requires the resonances to be formed earlier than the bulk hadrons, such that hadronic resonances can traverse the partonic medium.

\section{Resonance correlations in STAR}

A first attempt to study the high momentum charged hadron$\phi(1020)$ resonance correlation using the STAR detector was shown last year [12]. The new analysis presented in these proceedings is using $2.33 \mathrm{M}$ min bias $\mathrm{Cu}+\mathrm{Cu}$ events at $\sqrt{s_{N N}}=200 \mathrm{GeV}$. In the correlation analysis the trigger hadron is required to have a $p_{\mathrm{T}}>3 \mathrm{GeV} / c$ (2.58 M trigger) and an associated $\phi(1020)$ meson with $p_{\mathrm{T}}=1-2 \mathrm{GeV} / c$. This momentum range was chosen to select mostly events including only one trigger hadron. In the future we would like to place a higher momentum cut on the trigger hadron to select the more energetic jets. According to the formation time calculations one would prefer to select $\phi(1020)$ $\left(p_{\mathrm{T}}=(1-2) \mathrm{GeV} / c\right)$ from jet fragmentation between $p_{\mathrm{T}}=$ $3-10 \mathrm{GeV} / c$, but due to the lack of statistics in the available STAR dataset we are limited to a smaller momentum range and thus this study serves more as a proof of principle. The $\Delta \phi$ distribution between the trigger hadron and the associated $\phi(1020)$ meson is obtained by determining a yield from the invariant mass signal independently in each $\Delta \phi$ bin. As an example, Fig. 4.1 and Fig. 4.2 show the invariant mass distribution and extracted $\phi(1020)$ meson yield 
for the $\Delta \phi=[-1 / 18 \pi, 3 / 18 \pi]$ (same-side) peak bin and $\Delta \phi=[19 / 18 \pi, 23 / 18 \pi]$ (away-side) peak bin. The signal is shown after mixed event background subtraction. The uncorrelated background is generated with Kaon pairs where the two kaons are from different evens. The events however belong to the same event category described by the vertex position of the reaction, the reaction plane and the multiplicity.

The extracted yield for all $\Delta \phi$ angles is shown in Fig. 4.3. These data are not corrected for elliptic flow. The derived mass and width of the same-side signal (Fig. 4.1) is $\mathrm{m}=$ $1019.0 \pm 0.3 \mathrm{MeV} / \mathrm{c}^{2}$ and $\Gamma=6.0 \pm 0.7 \mathrm{MeV} / \mathrm{c}^{2}$ based on fit using a Breit-Wigner function combined with a linear background function. The derived mass and width of the away-side signal (Fig. 4.2) is $\mathrm{m}=1019.1 \pm 0.3 \mathrm{MeV} / \mathrm{c}^{2}$ and $\Gamma=7.2 \pm 0.9 \mathrm{MeV} / \mathrm{c}^{2}$. An additional systematic error of $2 \mathrm{MeV} / \mathrm{c}^{2}$ for the width depending on the fit range and normalization of the background needs to be applied in all $\Delta \phi$ bins. The derived mass and width values are in agree-

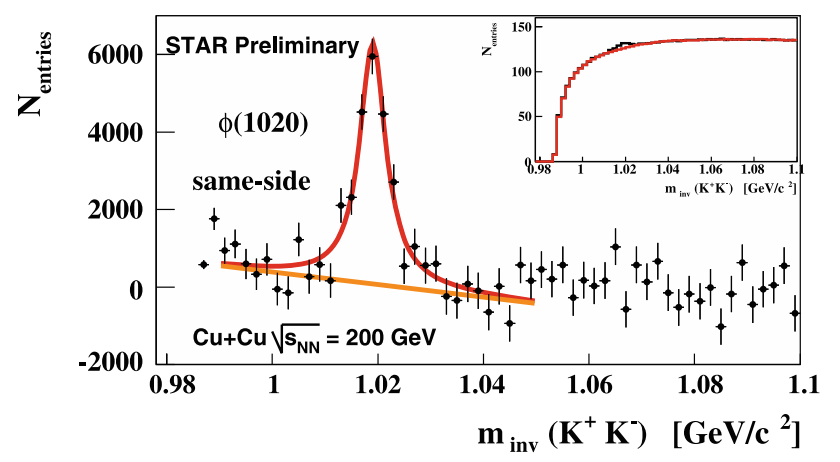

Fig. 4.1 Invariant mass distributions of $\phi(1020) \rightarrow K^{+}+K^{-}$ $\left(p_{\mathrm{T}}=(1-2) \mathrm{GeV} / c\right)$ for the in jet plane angular correlation (same-side) $\Delta \phi=[-1 / 18 \pi, 3 / 18 \pi]$ with respect to a $p_{\mathrm{T}}=$ (3-10) $\mathrm{GeV} / c$ hadron trigger in $\mathrm{Cu}+\mathrm{Cu}$ collisions at $\sqrt{s_{N N}}=200 \mathrm{GeV}$ before (inset) and after mixed-event background subtraction

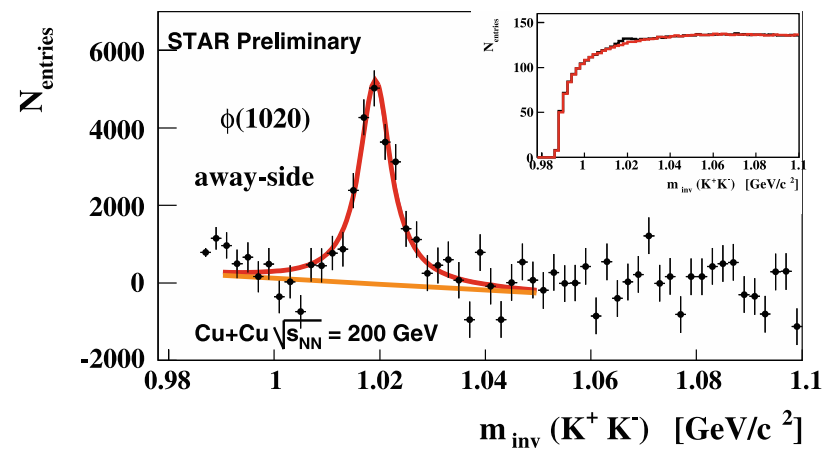

Fig. 4.2 Invariant mass distributions of $\phi(1020) \rightarrow K^{+}+K^{-}$ $\left(p_{\mathrm{T}}=(1-2) \mathrm{GeV} / c\right)$ for the in jet plane angular correlation (away-side) $\Delta \phi=[19 / 18 \pi, 23 / 18 \pi]$ with respect to a $p_{\mathrm{T}}=(3-10) \mathrm{GeV} / c$ hadron trigger in $\mathrm{Cu}+\mathrm{Cu}$ collisions at $\sqrt{s_{N N}}=200 \mathrm{GeV}$ before (inset) and after mixed-event background subtraction ment with the PDG values, when folded with the detector momentum resolution and energy loss in the detectors.

We are using the $\phi(1020)$ as the associated particle since its reconstructed mass spectrum has the largest significance of all short lived resonances in STAR. However the lifetime of the $\phi(1020)$ is about $45 \mathrm{fm} / c$, which means that the majority will decay outside of the medium. The $20 \mathrm{M}$ minimum bias $\mathrm{Cu}+\mathrm{Cu}$ events analyzed are not sufficient to place an effective high momentum cut on the $\phi(1020)$ spectrum. The analyzed data show no evidence for mass shifts and width broadenings of resonances in the momentum range of $p_{T}=(1-2) \mathrm{GeV} / c$, neither on the same side nor on the away side. This was to be expected since $\phi(1020)$ mesons in this momentum range are most likely dominated by interactions in the late hadronic phase and will subsequently decay outside of the fireball due to the long lifetime of the $\phi(1020)$. In addition the jet signal is sitting on a large background $(S / B=0.2)$. Therefore the invariant mass yield of the $\phi(1020)$ resonance in this analysis is dominated by nonjet triggered entries. To enhance the resonances from jets a higher momentum range is required.

Presently, the momentum of the kaon candidates for the $\phi(1020)$ reconstruction is restricted to $p_{\mathrm{T}}(0.2-1.1) \mathrm{GeV} / c$
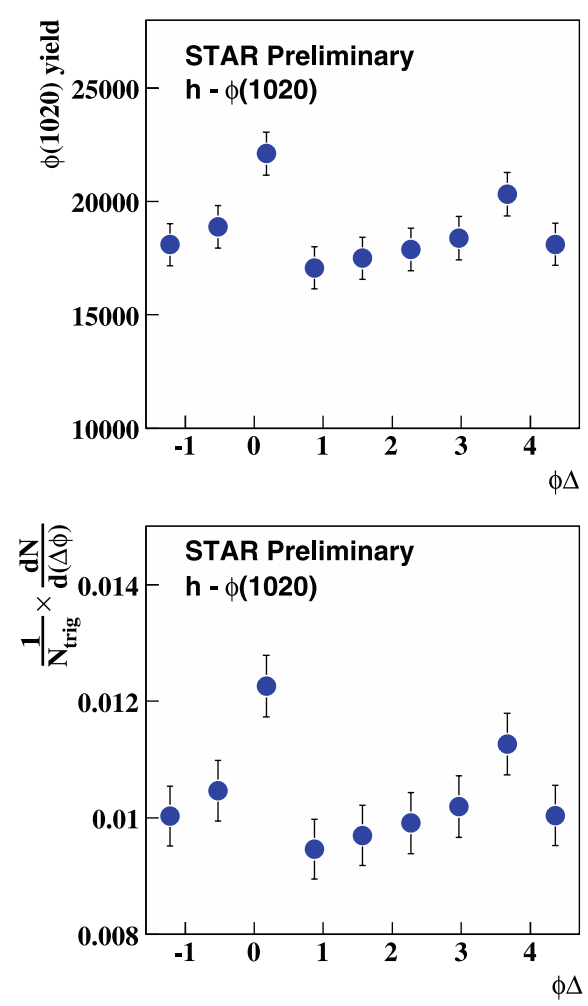

Fig. 4.3 Angular correlation of hadron- $\phi(1020)$ resonance. Hadron trigger $p_{\mathrm{T}}>3 \mathrm{GeV} / c$ and associated $\phi(1020) p_{\mathrm{T}}=(1-2) \mathrm{GeV} / c$. Upper: $\phi(1020)$ yield versus angle between $\phi(1020)$ and hadron trigger. Lower: Normalized by number of trigger hadrons. The errors are statistical only. The distribution is not corrected for elliptic flow $v_{2}$. The $y$-axis of the plots is zero suppressed 
in order to achieve clean particle identification in the TPC. In the future we will be able to select the higher momentum $\phi(1020)$ s with better significance by using the additional Time of Flight (TOF) detector $[13,14]$ which enables us to identify kaons up to $p=1.5-2.0 \mathrm{GeV} / c$.

The requirement that the resonance not only needs to form but also decay inside the medium, in order to probe chiral symmetry in the boosted laboratory frame, drives the need for very short lived resonances in this analysis. Therefore $K(892)$ or $\Delta(1232)$ resonances are more suited than, for example, the $\phi(1020)$. Nevertheless any additional medium modification might broaden the width and therefore shorten the lifetime, so that a $\phi(1020)$ might be still a good candidate [15]. The probability of the medium modified resonance decaying in the radially expanding plasma has only been qualitatively addressed at present. Calculations to determine the fraction of resonance decays in the medium are underway.

\section{Conclusions}

Heavy hadronic resonances from jets are suitable to study chiral symmetry restoration due to their short formation time. Theoretical calculations show that we expect short formation times of a few $\mathrm{fm} / \mathrm{c}$ for heavy mass hadrons up to $p_{\mathrm{T}}=10 \mathrm{GeV} / c$. An analysis technique based on jet correlations to enrich the resonances which pass through the medium has been proposed and also used to explore the phenomena in $\mathrm{Cu}+\mathrm{Cu}$ collisions. Future detector upgrades such as the TOF detector for STAR are necessary to significantly reduce the background in the reconstruction of hadronically decaying high momentum resonances.

\section{References}

1. P. Petreczky, Nucl. Phys. A 785, 10-17 (2007)

2. A. Adil, I. Vitev, hep-ph/0611109

3. C. Markert, R. Bellwied, I. Vitev, Phys. Lett. B 669, 92-97 (2008)

4. K. Gallmeister, T. Falter, Phys. Lett. B 630, 40 (2005)

5. B.I. Abelev et al. (STAR Collaboration), Phys. Rev. Lett. 97, 132301 (2006)

6. G. Torrieri et al., Phys. Lett. B 509, 239 (2001)

7. J. Rafelski et al., Phys. Rev. C 64, 054907 (2001)

8. J. Rafelski et al., Phys. Rev. C 65, 069902 (2002)

9. C. Markert et al., hep-ph/0206260

10. J. Adams et al., Phys. Rev. C 71, 044906 (2005)

11. M. Bleicher et al., Phys. Lett. B 530, 81 (2002) and private communication

12. C. Markert et al., in Proceedings of 23rd WWND. nucl-ex/ 0706.0724

13. B. Bonner et al., Nucl. Instrum. Methods A 508, 181 (2003)

14. M. Shao et al., Nucl. Instrum. Methods A 492, 344 (2002)

15. L. Holt, K. Haglin, J. Phys. G 31, S245 (2005) 